\title{
ChemComm
}

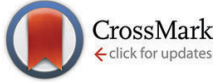

Cite this: Chem. Commun., 2014, 50, 9954

Received 14th May 2014

Accepted 5th July 2014

DOI: $10.1039 / c 4 c c 03656 e$

www.rsc.org/chemcomm

\section{An STM study of molecular exchange processes in organic thin film growth $\dagger$}

\author{
José M. Gallego, ${ }^{\text {ab }}$ David Ecija, ${ }^{a}$ Nazario Martín, ${ }^{\text {ac }}$ Roberto Otero ${ }^{\text {ad }}$ and \\ Rodolfo Miranda ${ }^{\text {ad }}$
}

The growth of a fullerene derivative (PCBM) on top of a layer of a tetrathiafulvalene (TTF) derivative previously deposited on $\mathrm{Au}(111)$ has been studied by scanning tunneling microscopy (STM). The results show that the preferential interaction with the gold substrate induces the exchange of PCBM molecules with the exTTF monolayer, expelling exTTF molecules to the outer surface. This exchange process is forbidden when the thickness of the exTTF layer increases above the monolayer, and the larger surface energy of PCBM leads to the growth of 3D islands.

As the field of organic electronics is evolving, it is becoming increasingly evident that the efficiency of the final devices is strongly dependent on the quality and the actual composition of the different interfaces within. ${ }^{1}$ The realization that small changes in the local environment of the interface at the molecular level can produce important changes in electronic and optical properties has prompted the study of both organic-inorganic ${ }^{2}$ and organic-organic heteroepitaxy. ${ }^{3}$ However, when compared to all-inorganic epitaxy, the field is still in its infancy: the existence of many additional degrees of freedom makes drawing general conclusions from a particular system very difficult. ${ }^{2 a, 4}$

Besides epitaxy, which strictly speaking refers only to the orientation and crystallinity of the growing layer with respect to the substrate, other factors that may affect the interface quality are the roughness and/or interdiffusion at the interface. ${ }^{2 a}$ Although roughness has been treated in some cases, ${ }^{5}$ there are very few reports on interdiffusion, ${ }^{6}$ and seldom at the molecular level.

In this manuscript, we report an STM study of the exchange processes that take place when PCBM (phenyl-C61-butyric acid

\footnotetext{
${ }^{a}$ Instituto Madrileño de Estudios Avanzados en Nanociencia (IMDEA-Nanociencia), E-28049 Madrid, Spain. E-mail: josemaria.gallego@imdea.org

${ }^{b}$ Instituto de Ciencia de Materiales de Madrid, CSIC, E-28049 Madrid, Spain

${ }^{c}$ Dpto. de Química Orgánica, Universidad Complutense de Madrid, E-28040 Madrid, Spain

${ }^{d}$ Dpto. de Física de la Materia Condensada, Universidad Autónoma de Madrid, E-28049 Madrid, Spain

$\dagger$ Electronic supplementary information (ESI) available: Additional STM images. See DOI: $10.1039 / \mathrm{c} 4 \mathrm{cc} 03656 \mathrm{e}$
}
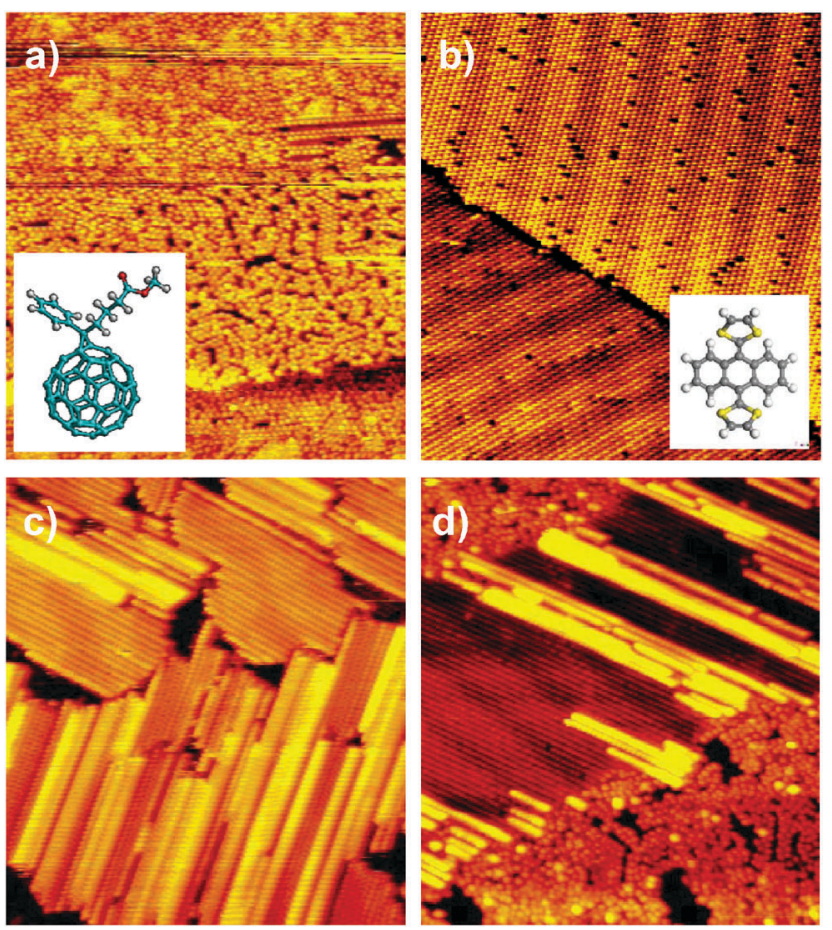

Fig. 1 STM images (59 $\mathrm{nm} \times 66 \mathrm{~nm}$ ) taken after depositing: (a) $1 \mathrm{ML}$ of PCBM on Au(111); (b) 1 ML of exTTF on Au(111); (c) $1.4 \mathrm{ML}$ of exTTF on Au(111); and (d) $\sim 0.6 \mathrm{ML}$ of exTTF on $\sim 0.5 \mathrm{ML}$ of PCBM previously grown on $\mathrm{Au}(111)$. (Measurement temperatures: (a) 300 K; (b) 150 K; (c) 300 K; and (d) 300 K.)

methyl ester, see the inset in Fig. 1a) is sublimated on top of a layer of a TTF derivative (2-[9-(1,3-dithiol-2-ylidene)anthracen10(9H)-ylidene]-1,3-dithiole, exTTF, see the inset in Fig. 1b) epitaxially grown on $\mathrm{Au}(111)$. By comparing with the previous studies on the growth of PCBM on $\mathrm{Au}(111)^{7}$ and exTTF on $\mathrm{Au}(111){ }^{8}$ the results presented here show how the deposition of PCBM partially transforms the exTTF/Au(111) into a PCBM/Au(111) interface by displacing a large fraction of the exTTF molecules to the outer surface.

PCBM, a fullerene derivative, is a well-known strong electron acceptor commonly used in bulk heterojunction solar cells. 
At room temperature the PCBM molecules are highly mobile on the $\mathrm{Au}(111)$ surface. If the substrate temperature is lowered to $150 \mathrm{~K}$, different structures are formed depending on the local coverage. Close to the monolayer, a disordered structure is found, which only exhibits a short-range hexagonal order (Fig. 1a). ${ }^{7}$ exTTF, on the other hand, is a relatively strong electron donor molecule. When deposited on $\mathrm{Au}(111)$ the exTTF molecules form striped islands composed of ordered arrays of 1D molecular rows that follow the compact direction of the substrate. Upon increasing the coverage, these islands coalesce, forming a number of alternating domains with different orientations and a typical width of $50 \mathrm{~nm}$ (Fig. 1b). The second and third layer growth also proceeds in the form of $1 \mathrm{D}$ rows in registry with the first monolayer, but their lateral arrangement lacks the long-range order observed for the first monolayer (see Fig. 1c).

The growth of mixed PCBM-exTTF 2D layers has also been reported. ${ }^{9}$ After depositing $\sim 0.5 \mathrm{ML}$ of exTTF on top of an already grown 0.5 ML of PCBM, the STM images (Fig. 1d) show that exTTF and PCBM do not mix; in contrast, exTTF disturbs the PCBM arrangement to form elongated islands similar to the ones formed in the absence of PCBM, confining PCBM to the space between the islands. The amount of exTTF exceeding the available free space grows in the form of second layer on top of the exTTF islands. A similar result is obtained if exTTF is deposited before PCBM. ${ }^{9}$ The resulting morphology, a lateral superlattice of interdigitated nanoscale stripes with a characteristic width of about $10-20 \mathrm{~nm}$, has been predicted to optimize the efficiency of bulk-heterojunction organic solar cells. ${ }^{10}$

In an attempt to build an exTTF-PCBM vertical superlattice, we deposited PCBM on top of a complete monolayer of exTTF. The STM images taken immediately after depositing $\sim 0.15 \mathrm{ML}$ of PCBM (Fig. 2a) show that the PCBM molecules form small, disordered clusters over the exTTF layer. These clusters seem to nucleate preferentially, but not exclusively, on the domain frontiers of the exTTF layer. However, images taken $30 \mathrm{~min}$ after deposition, with the sample kept at $150 \mathrm{~K}$, show that the morphology of the second layer has changed substantially (Fig. 2b). Instead of forming disordered clusters, the second layer molecules form 1D rows in registry with the first layer exTTF rows, exactly as the second layer exTTF molecules do. Also, the slightly elongated shape of second layer molecules bears a strong resemblance with the shape of second layer molecules in exTTF-only films (see Fig. S2 in the ESI $\dagger$ ). The possibility of being second layer PCBM molecules epitaxially grown on the exTTF layer (it has been reported that $\mathrm{C}_{60}$ can grow epitaxially on other organic systems $)^{11}$ can be excluded because the separation between molecules along the rows is $7.7 \AA$ (as in $\mathrm{C}_{60}$, the minimum separation between two PCBM molecules is $10 \AA$ ). Moreover, inspection of the first molecular layer shows the appearance of embedded clusters of molecules, larger in size than exTTF molecules, and with a short range hexagonal order (see the circled areas in Fig. 2b and d; Fig. S3, ESI $\dagger$ ). We thus conclude that PCBM molecules initially on top of the exTTF layer are now in direct contact with the $\mathrm{Au}(111)$ surface in the first layer, while some exTTF molecules have been displaced from the first to the second layer. That is, PCBM molecules have exchanged places with the exTTF molecules underneath, which have, in turn, diffused over the
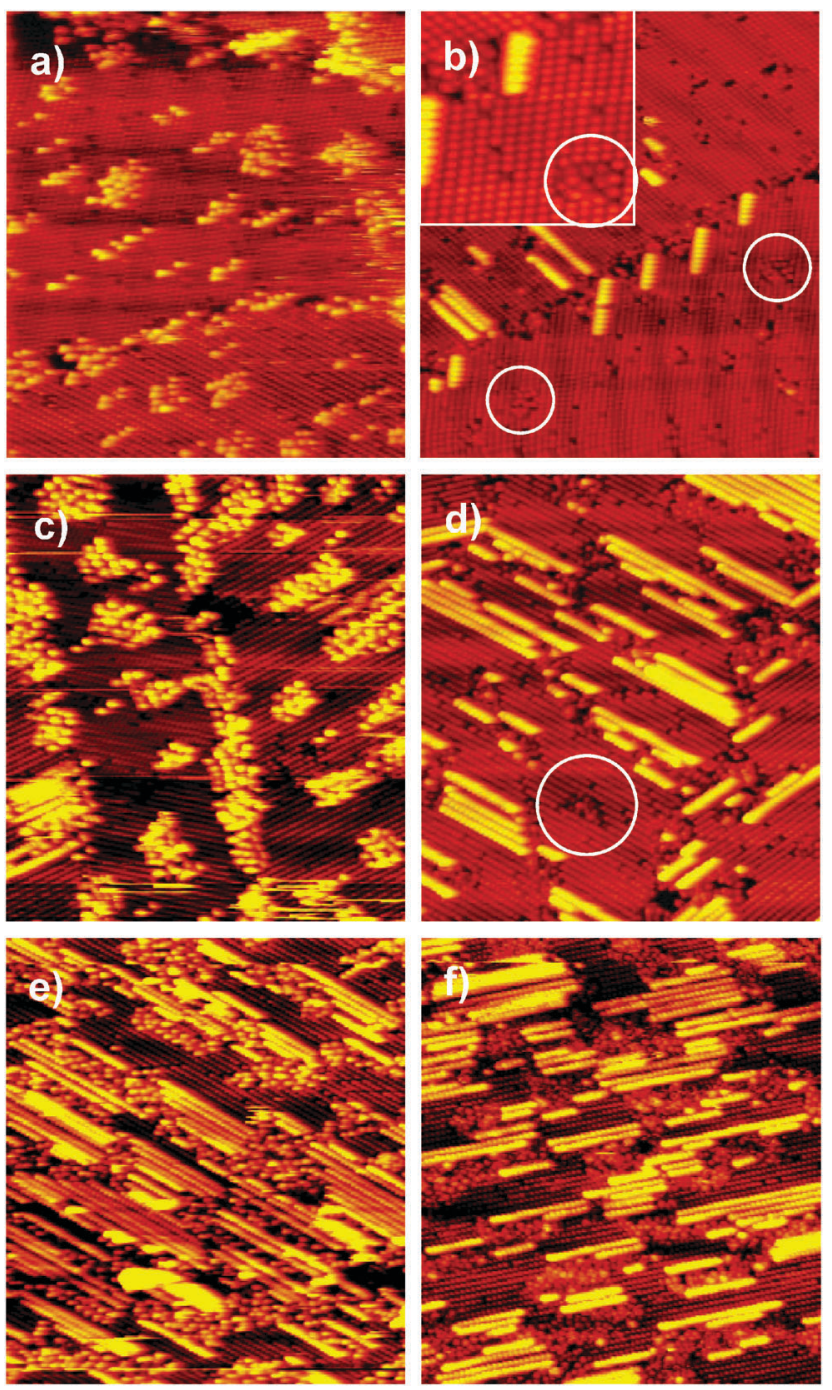

Fig. 2 (left) STM images (59 $\mathrm{nm} \times 66 \mathrm{~nm}$ ) of the $A u(111)$ surface taken immediately after depositing (a) $0.15 \mathrm{ML}$; (c) $0.30 \mathrm{ML}$; and (e) $0.45 \mathrm{ML}$ of PCBM on a complete layer of exTTF; (right) STM images of the samples shown in the left panel but taken $\sim 30$ minutes after deposition. (Measurement temperature: $150 \mathrm{~K}$.)

surface to form the characteristic second layer rows. Please note that, although both exTTF and PCBM were deposited with the substrate held at room temperature, STM measurements were carried out at $150 \mathrm{~K}$. At this temperature, the exTTF layer is almost completely frozen, as evidenced by the sequence imaging of the same area of the sample: the naturally occurring vacancies present in the exTTF layer and visible as dark spots in Fig. 1b do not show any displacement from one image to the next.

Similar results are obtained for higher PCBM coverages. The STM image (Fig. 2c) taken after depositing $\sim 0.15 \mathrm{ML}$ of PCBM on top of the previous sample shows the same disordered clusters of molecules. Once again, after a similar interval of time, the PCBM molecules exchange places with the exTTF molecules, going inside the exTTF layer. Correspondingly, the amount of exTTF now in the second layer is, within the error limits, equal to the total amount of deposited PCBM (Fig. 2d). 
For larger coverages (using the same deposition rate), the deposition time was large enough so that the number of exchange processes that had already taken place when the STM measurement started was already significant. The STM image shown in Fig. 2e was taken after depositing $\sim 0.45 \mathrm{ML}$ of PCBM $(120 \mathrm{~min})$ on the exTTF layer. The first measurements already show a mixed second layer composed of exTTF and PCBM molecules; but also in this case, after 30-40 minutes, the exchange process was complete (Fig. 2f), and the second layer was exclusively composed of exTTF molecules.

Although the influence of the scanning tip on the dynamics of the exchange processes cannot be completely ruled out, we can certainly exclude it as the main driving force: in every case, 30-40 minutes after deposition, all the second layer molecules were exTTF, and PCBM could only be found in the first layer, even when moving to a new, fresh region of the sample.

Place exchange is fairly common in metal-on-metal epitaxy, especially in fcc (100) and (110) surfaces, ${ }^{12}$ where it seems to be related to the atomistic mechanism of surface diffusion. ${ }^{13}$ These processes can lead to surface alloying, even for metals almost immiscible in bulk, ${ }^{14}$ or to the formation of islands of the deposited material within the first surface layer. ${ }^{12 d}$ The driving force for surface exchange processes has been attributed to different factors, such as a higher surface energy of the deposited material, ${ }^{15}$ a negative interfacial energy that would favour interfacial mixing, ${ }^{12 b}$ or to the strain caused by the atomic size mismatch, which can be considerably reduced by surface-confined mixing. ${ }^{16}$ In many cases, exchange processes can lead to surfactant-assisted epitaxial growth, since the floating layer can promote epitaxial growth by modifying the diffusion mechanism of the growing layer. ${ }^{13,17}$

The place exchange at the level of the monolayer is driven by the difference in the interaction with the $\mathrm{Au}(111)$ substrate; while the adsorption energy of exTTF on $\mathrm{Au}(111)$ is only of $0.32 \mathrm{eV}$ per molecule, ${ }^{8}$ the expected adsorption energy of PCBM is substantially larger, since for $\mathrm{C}_{60}$ on $\mathrm{Au}(111)$ it is of the order of $1.9 \mathrm{eV}$ per molecule. ${ }^{18}$ In order to determine the dependence of the exchange process on the thickness of the exTTF layer, we deposited $\sim 0.5 \mathrm{ML}$ of PCBM on top of a $\sim 2.5$ ML-thick film of exTTF (Fig. 3a). The STM image (Fig. 3b) seems to indicate that the PCBM molecules were not able to diffuse under the exTTF layer. Close-up images (see Fig. S4 in the ESI $\dagger$ ) show that the exTTF layer remains undistorted. The PCBM molecules diffused to the domain frontiers forming large 3D clusters, with an average lateral size of $\sim 50 \AA$ and an apparent height of $\sim 16 \AA$. This type of growth is commonly known as "Volmer-Weber", or island growth, and takes place when the atoms (or molecules) of the deposited material (PCBM) are more strongly bound to each other than to the substrate (exTTF). ${ }^{19}$ The requirement for island growth can also be expressed, in terms of the surface free energy $\gamma$, as $\gamma_{\text {exTTF }}<\gamma^{*}+\gamma_{\text {PCBM }}$, where $\gamma^{*}$ is the interfacial energy, ${ }^{20}$ which suggests that the surface free energy of a PCBM layer and/or the interfacial energy are larger than the one of exTTF. The surface free energies are proportional to the cohesive energies and the observation of 3D growth of PCBM on exTTF is in agreement with values for the cohesive energy of PCBM $(1.3 \mathrm{eV})^{21}$ and of the parent compound TTF $(\sim 1 \mathrm{eV}) .{ }^{22}$ With the above values, PCBM molecules are much more strongly bound to the gold substrate than to each other and, accordingly, the growth of PCBM
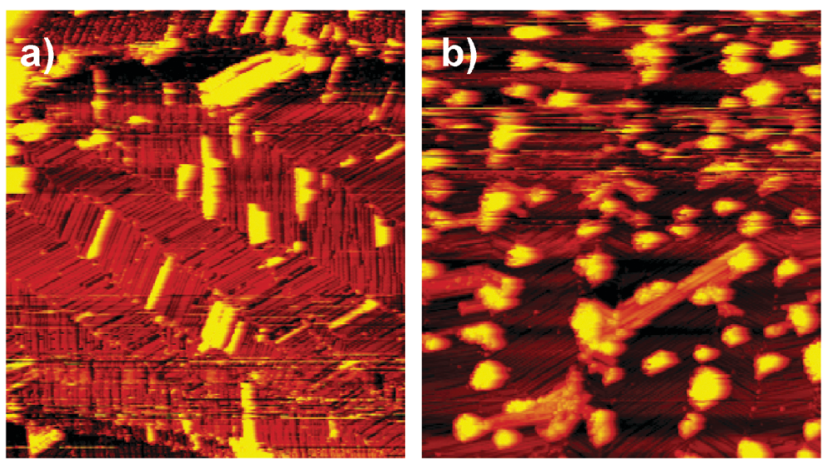

Fig. 3 (a) STM image $(236 \mathrm{~nm} \times 264 \mathrm{~nm})$ of $\sim 2.5 \mathrm{ML}$ of exTTF on the $\mathrm{Au}(111)$ surface; (b) STM image $(236 \mathrm{~nm} \times 264 \mathrm{~nm})$ taken after depositing $\sim 0.5 \mathrm{ML}$ of PCBM on the sample in (a). (Measurement temperature: $300 \mathrm{~K}$.)

on $\mathrm{Au}(111)$ is not of the island growth type, as shown in Fig. 1a. Then, the high energetic cost of having a flat PCBM layer on top of an exTTF layer can be avoided either by forming PCBM 3D clusters, or by binding to the gold layer. When grown on $1 \mathrm{ML}$ of exTTF, the PCBM molecules can exchange places with the exTTF molecules to bind strongly to $\mathrm{Au}(111)$, but $2 \mathrm{ML}$ of exTTF already constitute a barrier large enough to prevent the diffusion of PCBM to the gold layer (as Fig. 3 seems to indicate) and 3D island growth takes over.

On the other hand, despite the relatively strong $\pi-\pi$ interactions between individual fullerenes and exTTF derivatives, ${ }^{23}$ PCBM and exTTF do not mix on the gold surface (as shown in Fig. 1d), one of the reasons probably being that they are shape and structurally unequivalent, a condition that disfavours intermixing. ${ }^{3,24}$ So, in principle, if it were not for the different interaction with the gold substrate, the situation depicted in the right panel of Fig. 2 would be energetically unfavourable compared with the one shown in the left panel. In addition, the unit cell of the exTTF layer is rhombohedral, with sides 10.3 and $7.7 \AA$ in length forming an angle of $65^{\circ}$. On the other hand, the van der Waals radius of $\mathrm{C}_{60}$ is $\sim 10.0 \AA$. Thus, the simple exchange of a PCBM molecule with an exTTF molecule implies a strong distortion of the exTTF lattice (as can be seen in Fig. 2), with the implicit energy cost. This makes the exchange processes described here different from those previously reported related to alloying, since in the present case it is the presence of a third material, the gold surface, which is ultimately responsible for the intermixing between PCBM and exTTF.

In summary, we have shown that when PCBM is deposited on top of an exTTF monolayer previously adsorbed on $\mathrm{Au}(111)$, there are exchange processes between the PCBM and exTTF molecules, the former ending up embedded within the exTTF layer. These exchange processes are related to the larger adsorption energy of PCBM on the Au(111) layer. The exchange processes are limited to the first monolayer, as the higher surface free energy of PCBM takes over and leads to the growth of 3D islands of PCBM on exTTF for larger thickness.

Our work was supported by the MICINN of Spain (FIS201018847, FIS2012-33011), Comunidad de Madrid (Nanobiomagnet S2009/MAT-1726), CONSOLIDER-INGENIO on Molecular Nanoscience (CSD2007-00010) and European Union (SMALL PITN-GA2009-23884). 


\section{Notes and references}

1 (a) C.-a. Di, Y. Liu, G. Yu and D. Zhu, Acc. Chem. Res., 2009, 42, 1573; (b) A. Tada, Y. Geng, M. Nakamura, Q. Wei, K. Hashimoto and K. Tajima, Phys. Chem. Chem. Phys., 2012, 14, 3713; (c) N. R. Armstrong, W. Wang, D. M. Alloway, D. Placencia, E. Ratcliff and M. Brumbach, Macromol. Rapid Commun., 2009, 30, 717; (d) N. Koch, ChemPhysChem, 2007, 8, 1438.

2 (a) F. Schreiber, Phys. Status Solidi A, 2004, 201, 1037; (b) S. R. Forrest, Chem. Rev., 1997, 97, 1793.

3 A. Hinderhofer and F. Schreiber, ChemPhysChem, 2012, 13, 628.

4 W. Chen, D.-C. Qi, H. Huang, X. Gao and A. T. S. Wee, Adv. Funct. Mater., 2011, 21, 410.

5 (a) X. Zhang, E. Barrena, D. Goswami, D. G. de Oteyza, C. Weis and H. Dosch, Phys. Rev. Lett., 2009, 103, 136101; (b) H. Zhu, Q. L. Li, X. J. She and S. D. Wang, Appl. Phys. Lett., 2011, 98, 243304; (c) G. Hlawacek, P. Puschnig, P. Frank, A. Winkler, C. AmbroschDraxl and C. Teichert, Science, 2008, 321, 108.

6 (a) R. Ye, M. Baba, K. Suzuki and K. Mori, Appl. Surf. Sci., 2008, 254, 7885; (b) L. Sun, C. Liu, D. Queteschiner, G. Weidlinger and P. Zeppenfeld, Phys. Chem. Chem. Phys., 2011, 13, 13382.

7 D. Écija, R. Otero, L. Sánchez, J. M. Gallego, Y. Wang, M. Alcamí, F. Martín, N. Martín and R. Miranda, Angew. Chem., Int. Ed., 2007, 46, 7874 .

8 C. Urban, D. Ecija, Y. Wang, M. Trelka, I. Preda, A. Vollmer, N. Lorente, A. Arnau, M. Alcami, L. Soriano, N. Martin, F. Martin, R. Otero, J. M. Gallego and R. Miranda, J. Phys. Chem. C, 2010, 114, 6503.

9 R. Otero, D. Ecija, G. Fernandez, J. M. Gallego, L. Sanchez, N. Martin and R. Miranda, Nano Lett., 2007, 7, 2602.

10 J. Nelson, Curr. Opin. Solid State Mater. Sci., 2002, 6, 87.

11 (a) K. Itaka, M. Yamashiro, J. Yamaguchi, M. Haemori, S. Yaginuma, Y. Matsumoto, M. Kondo and H. Koinuma, Adv. Mater., 2006, 18, 1713; (b) A. Hinderhofer, A. Gerlach, K. Broch, T. Hosokai, K. Yonezawa, K. Kato, S. Kera, N. Ueno and F. Schreiber, J. Phys. Chem. C, 2012, 117, 1053; (c) L. Sanchez, R. Otero, J. M. Gallego, R. Miranda and N. Martin, Chem. Rev., 2009, 109, 2081.

12 (a) O. S. Hernan, A. L. V. de Parga, J. M. Gallego and R. Miranda, Surf. Sci., 1998, 415, 106; (b) H. Röder, R. Schuster, H. Brune and K. Kern, Phys. Rev. Lett., 1993, 71, 2086; (c) L. P. Nielsen, F. Besenbacher,
I. Stensgaard, E. Laegsgaard, C. Engdahl, P. Stoltze, K. W. Jacobsen and J. K. Norskov, Phys. Rev. Lett., 1993, 71, 754; (d) P. W. Murray, S. Thorshaug, I. Stensgaard, F. Besenbacher, E. Lægsgaard, A. V. Ruban, K. W. Jacobsen, G. Kopidakis and H. L. Skriver, Phys. Rev. B: Condens. Matter Mater. Phys., 1997, 55, 1380; (e) F. Nouvertné, U. May, M. Bamming, A. Rampe, U. Korte, G. Güntherodt, R. Pentcheva and M. Scheffler, Phys. Rev. B: Condens. Matter Mater. Phys., 1999, 60, 14382; $(f)$ V. Blum, C. Rath, S. Muller, L. Hammer, K. Heinz, J. M. Garcia, J. E. Ortega, J. E. Prieto, O. S. Hernan, J. M. Gallego, A. L. V. de Parga and R. Miranda, Phys. Rev. B: Condens. Matter Mater. Phys., 1999, 59, 15966.

13 J. Ferron, L. Gomez, J. M. Gallego, J. Camarero, J. E. Prieto, V. Cros, A. L. V. de Parga, J. J. de Miguel and R. Miranda, Surf. Sci., 2000, 459, 135.

14 A. Davies, J. A. Stroscio, D. T. Pierce and R. J. Celotta, Phys. Rev. Lett., 1996, 76, 4175.

15 J. A. Meyer, I. D. Baikie, E. Kopatzki and R. J. Behm, Surf. Sci., 1996, 365, L647.

16 J. Tersoff, Phys. Rev. Lett., 1995, 74, 434.

17 J. Camarero, J. Ferron, V. Cros, L. Gomez, A. L. V. de Parga, J. M. Gallego, J. E. Prieto, J. J. de Miguel and R. Miranda, Phys. Rev. Lett., 1998, 81, 850.

18 I. Hamada and M. Tsukada, Phys. Rev. B: Condens. Matter Mater. Phys., 2011, 83, 245437.

19 J. A. Venables, G. D. T. Spiller and M. Hanbucken, Rep. Prog. Phys., 1984, 47, 399.

20 J. A. Venables, Surf. Sci., 1994, 299-300, 798.

21 J. M. Nápoles-Duarte, M. Reyes-Reyes, J. L. Ricardo-Chavez, R. GaribayAlonso and R. López-Sandoval, Phys. Rev. B: Condens. Matter Mater. Phys., 2008, 78, 035425.

22 D. J. Sandman, A. J. Epstein, J. S. Chickos, J. Ketchum, J. S. Fu and H. A. Scheraga, J. Chem. Phys., 1979, 70, 305.

23 (a) D. Canevet, E. M. Pérez and N. Martín, Angew. Chem., Int. Ed., 2011, 50, 9248; (b) F. G. Brunetti, J. L. Lopez, C. Atienza and N. Martin, J. Mater. Chem., 2012, 22, 4188.

24 (a) A. Opitz, J. Wagner, W. Brütting, A. Hinderhofer and F. Schreiber, Phys. Status Solidi A, 2009, 206, 2683; (b) I. Salzmann, S. Duhm, R. Opitz, R. L. Johnson, J. P. Rabe and N. Koch, J. Appl. Phys., 2008, 104; (c) J.-O. Vogel, I. Salzmann, S. Duhm, M. Oehzelt, J. P. Rabe and N. Koch, J. Mater. Chem., 2010, 20, 4055. 